\title{
Saving our newborns by doing the basics right - and keeping it simple
}

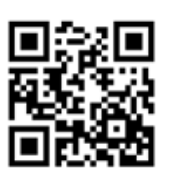

Just basic interventions could reduce South Africa (SA)'s neonatal death rate by up to $90 \%$, according to a world-acclaimed innovator in cheap, child-friendly therapies, Prof. Heather Zar, chief of Paediatrics and Child Health at Red Cross War Memorial Children's Hospital in Cape Town.

In view of the absence of significant reduction in SA's neonatal mortality rate over the past decade and the country failing dismally to reach this year's Millennium Development Goal (MDG) of reducing its 1990 child mortality rate by two-thirds, Izindaba quizzed Zar on the most vital interventions required.

Antenatal steroids to mature a baby's lungs when its mother is in preterm labour, the now world-famous kangaroo care, exclusive breastfeeding, optimal labour and delivery management, neonatal resuscitation and optimal postnatal care including appropriate treatment of neonatal sepsis would, taken together, send the neonatal death rate plummeting, she said.

In preventing preterm delivery, better antenatal and obstetric care would also go a long way to ensure safer delivery and effective subsequent postnatal care, she added. When it came to reducing under-5 mortality (another MDG SA didn't achieve), several well-known effective preventive strategies such as immunisation, optimising nutrition, breastfeeding, provision of antiretroviral therapy and prevention of motherto-child transmission (PMTCT) of HIV were required. As neonatal deaths make up almost a third of under- 5 deaths, strategies to reduce deaths in the first 28 days of life (and especially in the first 7 days) could have a big impact on the under-5 mortality rate.

\section{Where we have done \\ well ...}

The above are all areas in which SA has improved by varying degrees, especially PMTCT, where major reductions in HIV infections have for the first time put almost zero transmission within reach, achieving world acclaim. Vaccines for children were a growing local success story, with rates of invasive pneumococcal disease - including cases caused by antibiotic-resistant bacteria having fallen substantially following the introduction of a pneumococcal conjugate vaccine. The rate of infections resistant to two different antibiotics had declined nearly twice as much as that for infections that could be treated with antibiotics. This proportionately greater effect of vaccination on antibiotic-resistant strains pointed to a very valuable added benefit of immunisation. Zar said that a maternal influenza vaccine during pregnancy had also recently been shown to substantially reduce influenza in mothers and their babies, and was a strategy that should be strengthened as a matter of urgency. Despite official and scientifically backed recommendations for the influenza vaccine to be prioritised for pregnant women, uptake remained very low.

Zar described as 'very exciting' current overseas work on the development of a vaccine for respiratory syncytial virus (RSV), which causes infections of the lungs and respiratory tract. RSV is so common that most children have been infected with it by age 2 . In adults and older, healthy children, the symptoms of respiratory syncytial virus are mild and typically mimic the common cold. Self-care measures are usually all that are needed to relieve any discomfort, but infection can be severe in in premature babies and infants with underlying health conditions (and in older adults, adults with heart and lung diseases, or anyone with a very weak immune system). Zar said that a major part of her enthusiasm was due to the strategy to immunise pregnant women against RSV as a way of protecting their infants.

Overall, she said, strengthening health systems, reducing poverty and improving the coverage and uptake of these known effective interventions would see SA beginning to make quicker, long-awaited progress in reducing the neonatal mortality and under-5 mortality (and morbidity) rates.

\section{Leading by example with innovation}

Zar, a past president of the South African Thoracic Society and president of the Pan African Thoracic Society, currently chairs the Forum of the International Respiratory Societies. She achieved world acclaim with her low-cost plastic cooldrink bottle spacer for asthmatic children, which rendered what used to be expensive inhalers accessible to millions of children in poorer communities. Her invention made the oral alternative therapy, theophylline (a white crystalline alkaloid and vasodilator with several unpleasant side-effects), a thing of the past. The use of cooldrink bottles as asthma spacers is now included in guidelines from the Global Initiative for Asthma and the World Health Organization (WHO). Another surprisingly simple advance that she and her Red Cross War Memorial Hospital team came up with now also included in WHO guidelines and national recommendations - is the use of 
less invasive sputum induction for rapid tuberculosis (TB) testing in children. A few years ago it was widely believed that children couldn't provide enough sputum for a TB test. Instead doctors used gastric lavage or stomach pumping, a process that involved starving a child overnight, then sticking a tube down their nostrils or throat into their stomach, and sucking all the contents out. Children were subjected to this process three days in a row before doctors could retrieve a specimen large enough for testing. Zar and her team found that by nebulising a child beforehand and suctioning the back of their throat very quickly, it is possible to induce enough sputum for a rapid TB test (using
GeneXpert). This retrieves a better specimen than that from three days of gastric irrigation.

\section{Chris Bateman}

chrisb@hmpg.co.za

S Afr Med J 2015;105(3):247-248.

DOI:10.7196/SAMJ.955 